\title{
Fine-Tuning the Truth
}

\author{
Elisabeth E. Elder • Raimund Jakesz
}

Published online: 18 August 2011

(c) Société Internationale de Chirurgie 2011

Communication has become a major issue in cancer care, and rightly so. Good communication helps patients and their families understand the situation and assists with decision-making, acceptance, adjustment, and coping. It is well known that the way in which a cancer diagnosis is delivered not only affects the person's understanding of the illness, it can also have an impact on their longer-term psychological adjustment. While these issues are essential to medicine in general, they are particularly important for patients with cancer or other life-threatening conditions. Some unique aspects for breast cancer patients include the effects on women's sexuality and body image, fertility, and issues around premature menopause induced by adjuvant systemic therapy. Effective communication seems especially important for this latter group in which the high percentage of noncompliance seems to be based on inadequate communication [1]. In the "Gathering information of adjuvant endocrine therapy (GAEA) study," $41 \%$ of participating patients were not included in the decisionmaking process at the beginning of adjuvant treatment [2].

In response to these concerns, a plethora of communication training skill courses has become available and participation in such workshops constitutes part of the core curriculum for the training of cancer specialists in many countries [3]. Good communication skills are also very important tools of the surgeon, and development of these

E. E. Elder $(\bowtie)$

Westmead Breast Cancer Institute, Westmead Hospital, P.O. Box 143, Westmead, Sydney, NSW 2145, Australia

e-mail: e.elder@bigpond.net.au

\section{R. Jakesz}

Klinische Abteilung für Allgemeinchirurgie, Medizinische Universität Wien, Vienna, Austria

e-mail: raimund.jakesz@meduniwien.ac.at skills should not be reserved for only medical specialists and specialized nursing staff. It is obvious that surgery is not just about cutting! [4, 5].

In this issue of World Journal of Surgery, Mendick et al. [6] have published a study with the pertinent title "Telling everything, but not too much". It highlights the multilayered nature of the encounter between surgeon and patient and the range of functions this serves, in addition to simply conveying objective information. The study is particularly interesting as it contains information from both the doctor's and the patient's perspective. It describes through in-depth interviews what seems to be a very successful breast cancer care unit where the clinicians in general are able to meet the communication needs of their patients and target their approach accurately to the individual patient.

It is striking how similarly the surgeons and the patients described their communication goals: to give hope, to show surgeon's expertise (i.e., trust), to sustain a personal clinical relationship, and to support decision-making. Furthermore, the article emphasizes the complexity of the surgeon's task. Hope needs to be conveyed while maintaining honesty and not withholding any information, and the surgeon is expected to demonstrate authority while building up a personal relationship and to involve the patient in the decision-making process while taking responsibility for important decisions.

To achieve this, the surgeons selected and shaped the information and patients relied on them to do so. The necessity of this comes as no surprise to most experienced clinicians. To simply give all available information without taking the individual's needs and state of mind into consideration can easily lead to information overload and confusion. Although it is clear that contemporary Western ethics emphasize the right of the cancer patient to be fully informed and to participate in decisions about treatment, 
patients vary in the extent to which they want to be involved, and may express different choices to different members of the multidisciplinary team and may vary their choices from time to time. There are also important cultural, social, and religious differences, and sometimes language barriers become an issue. We believe that the key to balancing these objectives is to listen actively, to give the patient time, not to make assumptions, and not to have expectations about the way we would like a patient to respond.

It is interesting that the top priorities were to convey and sustain hope. The vital importance of hope to counterbalance the risk of depression has become increasingly evident to health professionals. After a period of focusing on technical advances, there is now a resurgence of interest in improving patient adjustment and coping by emphasizing hope and self-empowerment. The benefits of improved coping may also extend beyond the psychological realm. Meta-analysis of studies evaluating stress-associated psychosocial factors and prognosis in patients with malignant disease clearly shows that there is a correlation between stressful events and inadequate strategies for coping with painful emotional reactions and impaired quality of life and increased mortality [7]. In further studies, when compared to assessment only, psychosocial intervention provided by weekly 90-min group sessions over 18 weeks has been associated with increased recurrence-free and overall survival in the adjuvant stage and increased cumulative survival after recurrence [8, 9]. So, as opposed to the traditional medical view in which the doctor provides a cure, the patient herself may wish to play an important, active role that we should support; a woman can be strengthened by believing she can do "something" for herself rather than passively letting things (treatment) happen.

Some say that understanding that diseases play an important role in our lives constitutes an important message from our psyche and that this needs to be addressed. Perhaps we should provide an opportunity for patients to look at their disease in that way, led by their physician or other members of the multidisciplinary team. The question remains how actively should we train the patient to get involved in the body-mind interaction: should we teach our patients meditation? Not all of us will feel comfortable dealing with these issues, so it may be important for surgeons to receive practical instruction and supervision along the lines of: How should I do it? We also need to understand that we can give only what we have; in other words, we also need to look at our own personal (spiritual) evolution and how we deal with challenging events in our lives.

Clinical practice guidelines for the psychosocial care of breast and other types of cancer patients as well as guidelines for communicating bad news have been issued by a number of international bodies [10-12]. In their article, Mendick et al. [6] criticize communication advice in general as being linear and not reflecting clinical practice. We agree that for any guidelines to be endorsed they need to be practical and feel relevant to the clinicians. However, communication guidelines should not be seen as cookbooks but rather as ways to increase awareness and stimulate us reflect on appropriate communication styles. With that in mind, we can aim to balance reassurance with truthful disclosure, being clear without being patronizing, and displaying empathy rather than detachment. It would seem difficult to formulate universal guidelines that exactly describe how to do this and how to sense who wants how much and what level of participation. Maybe this is where the art of being a good doctor comes in.

\section{References}

1. Davidson B, Vogel V, Wickerham L (2007) Oncologist-patient discussion of adjuvant hormonal therapy in breast cancer: results of a linguistic study focusing on adherence and persistence to therapy. J Support Oncol 5:139-145

2. Wengström Y, Aapro M, Leto di Priolo S et al (2007) Patients' knowledge and experience of adjuvant endocrine therapy for early breast cancer: a European study. Breast 16(5):462-468

3. Stiefel F, Barth J, Bensing J et al (2010) Communication skills training in oncology: a position paper based on a consensus meeting among European experts in 2009. Ann Oncol 21(2): 204-207

4. Mueller R (2010) ESTS Presidential address: the driving force. Eur J Cardiothorac Surg 38:651-656

5. Riegler FM (2011) Editorial: "it is all about our academy". Eur Surg 43(1): $1-4$

6. Mendick N, Young B, Holcombe C, Salmon P (2011) Telling 'everything' but not 'too much': the surgeon's dilemma in consultations about breast cancer. World J Surg 35. doi: 10.1007/s00268-011-1195-3

7. Chida Y, Hamer M, Wardle J et al (2008) Do stress-related psychosocial factors contribute to cancer incidence and survival? Nat Rev Clin Oncol 5:466-475

8. Andersen B, Farrar WB, Golden-Kreutz DM et al (2004) Psychological, behavioral, and immune changes after a psychological intervention: a clinical trial. J Clin Oncol 22(17):3570-3580

9. Andersen B, Thornton LM, Shapiro CL et al (2010) Biobehavioral, immune, and health benefits following recurrence for psychological intervention participants. Clin Cancer Res 16: 3270-3278

10. National Breast Cancer Centre, National Cancer Control Initiative (2003) Clinical practice guidelines for the psychosocial care of adults with cancer. Camperdown NSW, Australia. http://www. nhmrc.gov.au/guidelines/publications/cp90. Accessed Aug 2011

11. National Institute for Health and Clinical Excellence (2004) Improving supportive and palliative care for adults with cancer. http://www.nice.org.uk/CSGSP. Accessed Aug 2011

12. Turner J, Zapart S, Pedersen K et al (2005) Clinical practice guidelines for the psychosocial care of adults with cancer. Psychooncology 14(3):159-173 